\title{
SOLUTIONS OF THE EINSTEIN-MAXWELL-DIRAC AND SEIBERG-WITTEN MONOPOLE EQUATIONS
}

\author{
Cihan Saçlıoğlu ${ }^{1,2}$ \\ ${ }^{1}$ Physics Department, Bog̃aziçi University \\ 80815 Bebek-İstanbul, Turkey \\ and \\ ${ }^{2}$ Feza Gürsey Institute \\ TUBITAK-Bog̃aziçi University \\ 81220 Çengelköy, Istanbul- Turkey
}

\begin{abstract}
We present unique solutions of the Seiberg-Witten Monopole Equations in which the $U(1)$ curvature is covariantly constant, the monopole Weyl spinor consists of a single constant component, and the 4-manifold is a product of two Riemann surfaces of genuses $p_{1}$ and $p_{2}$. There are $p_{1}-1$ magnetic vortices on one surface and $p_{2}-1$ electric ones on the other, with $p_{1}+p_{2} \geq 2\left(p_{1}=p_{2}=1\right.$ being excluded $)$. When $p_{1}=p_{2}$, the electromagnetic fields are self-dual and one also has a solution of the coupled euclidean Einstein-Maxwell-Dirac equations, with the monopole condensate serving as cosmological constant. The metric is decomposable and the electromagnetic fields are covariantly constant as in the Bertotti-Robinson solution. The Einstein metric can also be derived from a Kähler potential satisfying the Monge-Ampère equations.
\end{abstract}




\section{Introduction:}

Four-dimensional manifolds of Euclidean signature are of interest in physics for semiclassical treatments of vacuum tunnelling and for their contributions to the path integral of Quantum Gravity. In such a physical context, one might start by trying to solve Einstein's equations in the presence of some chosen set of matter sources, together with field equations (adapted to the background metric) for the matter fields. In mathematics, on the other hand, four dimensions are unique in hosting infinitely many manifolds that are homeomorphic but not diffeomorphic to each other. The most efficient approach for classifying such manifolds involves the Seiberg-Witten monopole equations (SWME) []

$$
\begin{gathered}
\not D_{A} \psi=0, \\
F_{\mu \nu}^{+} \equiv \frac{1}{2}\left(F_{\mu \nu}+\frac{1}{2} \epsilon_{\mu \nu \alpha \beta} F^{\mu \nu}\right)=-\frac{i}{4} \psi^{\dagger}\left[\gamma_{\mu}, \gamma_{\nu}\right] \psi .
\end{gathered}
$$

In (11) and (2) $A_{\mu}$ is the $U(1)$ connection and $F_{\mu \nu}^{+}$is the self-dual part of $F_{\mu \nu}=$ $\partial_{\mu} A_{\nu}-\partial_{\nu} A_{\mu}$; the covariant Dirac operator also involves the spin connection of the 4-manifold $\mathcal{M}_{4}$. For a pedagogical review of the SWME, we refer the reader to [2]. The equations stem from an $N=2$ supersymmetric Yang-Mills theory which is first "twisted" into a topological quantum field theory (TQFT) [3] and then has its gauge symmetry broken down spontaneously from $S U(2)$ to $U(1)$. For the particular choice of Higgs vacuum used in the SWME, the classical monopole solutions, represented by the Weyl spinor $\psi$, become massless, which is why a 
mass term is absent in (11).

In the mathematical study of 4-manifolds, the focus is usually on global questions (e.g. topological invariants, the moduli space of a class of solutions etc.) rather than explicit local solutions of the SWME. However, it is instructive to derive from the SWME, in actual local form, a solution whose global properties are known. Another reason for searching for local solutions is the possibility that some of these may represent configurations of physical significance, which may then indicate new connections between the topology of 4-manifolds and physics. In particular, it is important to note that there are two SWME equations for three fields; the fact that the metric is not constrained by a third equation is of course consistent with the topological nature of the SW system. If one were to consider a "physical" equation for the metric, the obvious candidate would of course be Einstein's field equations in the presence of a Maxwell and a Dirac (or, rather, Weyl) field. To make the system completely physical, Maxwell's equations would also have to be added, resulting in an overdetermined system because of (2). LeBrun [4] has in fact recently considered manifolds simultaneously obeying the SWME and Einstein's equations with a cosmological term without the energymomentum tensors of the Maxwell and Dirac fields on the RHS; we will see in a specific example below how and when this can be justified.

Remarkably, a set of solutions to this overconstrained system exist as reported briefly in [5]. One of the purposes of the present paper is to exhibit their deriva- 
tion in detail; but we start with our more general SWME solutions of which the "physical" ones are a special subset: The 4-manifold $\mathcal{M}_{4}$ is a product of two Riemann surfaces $\Sigma_{p_{1}}$ and $\Sigma_{p_{2}}$, where the genuses $p_{1}$ and $p_{2}$ must satisfy $p_{1}+p_{2} \geq 2$, excluding $p_{1}=p_{2}=1$. The Weyl spinor $\psi$ consists of a single constant component $\psi_{1}$ or $\psi_{2}$, which may be interpreted as a monopole condensate. It is worth remarking that in the closely related "physical" Seiberg-Witten theory [6] based on an untwisted $N=2$ supersymmetric Yang-Mills theory in flat Minkowski space, precisely such a condensate leads to quark confinement, while a "gluino condensate", possibly dual to the monopole one, is considered in [3]. Physically, $p_{1}-1\left(p_{2}-1\right)$ is the number of magnetic (electric) vortices in $\Sigma_{p_{1}}\left(\Sigma_{p_{2}}\right)$.

Interestingly, it is the most symmetric special case (with self-dual electromagnetic fields) of the above SWME solutions that solves the coupled EinsteinMaxwell-Dirac equations, with the condensate now serving as the cosmological constant. The solution is reminiscent of the Bertotti-Robinson one [7], [8] in that the metric is decomposable and the electromagnetic fields are covariantly constant. However, the Euclidean signature and the spinor (not the cosmological constant, which is present in [7]) are new features. The presence of the spinor in particular provides a counterexample to the folk-theorem that one need not seek solutions of the coupled Einstein-Maxwell-Dirac equations since the Dirac field supposedly becomes negligible in the classical limit where the field equations apply. 
The plan of the paper is as follows. In section 2, we describe the Ansatz for the SWME, which are then reduced to a pair of Liouville equations. In section 3, we discuss the behavior of the Ansatz under gauge transformations. The solutions, which are expressed in terms of Fuchsian functions, are classified and presented in section 4. We compute the topological numbers of the solutions in section 5, and using these, prove their uniqueness by showing that the virtual dimension of the moduli space of solutions is zero. In section 6 , we show that the special self-dual SWME solution is the only one which also satisfies the Einstein-Maxwell-Dirac equations. In section 7, the Kähler form of the metric and the Monge-Ampère equation satisfied by the Kähler potential are given. Section 8 ends the paper with concluding remarks.

\section{An Ansatz based on $\mathcal{M}_{2}^{(1)} \times \mathcal{M}_{2}^{(2)}$ :}

A central tool in Seiberg-Witten theory of 4-manifolds is the Weitzenbock formula obtained by squaring $\not D_{A}$ in (1); together with (2), it can be shown to imply that 4-manifolds with everywhere non-negative scalar curvatures cannot admit non-singular solutions. Integrating the same formula, Witten derived "vanishing theorems" showing that the solutions for non-negative curvature are not only singular, but also non-square integrable. Thus we expect that at least one of the two Riemann surfaces will have genus $p \geq 2$; if one surface is the two-sphere, the curvature of the higher genus surface must be sufficiently large and negative to make the overall scalar curvature negative. This expectation will be explicitly 
verified by the computation that follows.

We start with the conformally flat basis 1 -forms

$$
e^{i}=e^{\mu} d x^{i}, \mu=\mu\left(x^{1}, x^{2}\right), i=1,2 ; \quad e^{j}=e^{\nu} d x^{j}, \nu=\nu\left(x^{3}, x^{4}\right), j=3,4
$$

for $\mathcal{M}_{2}^{(1)}$ and $\mathcal{M}_{2}^{(2)}$, respectively. We also choose the similarly "decomposable" $U(1)$ connection

$$
A_{\mu}=\left(A_{1}\left(x^{1}, x^{2}\right), A_{2}\left(x^{1}, x^{2}\right), A_{3}\left(x^{3}, x^{4}\right), A_{4}\left(x^{3}, x^{4}\right)\right)
$$

while the spinor $\psi^{T}=\left(\psi_{1}, \psi_{2}, 0,0\right)$ is initially assumed to depend on all four coordinates. The Cartan structure equations yield the spin-connection 1-forms

$$
\omega^{12}=\partial_{2} \mu d x^{1}-\partial_{1} \mu d x^{2}, \omega^{34}=\partial_{4} \nu d x^{3}-\partial_{3} \nu d x^{4}
$$

with all other components vanishing. Of the curvature 2 -forms

$$
R_{b}^{a}=d \omega_{b}^{a}+\omega_{c}^{a} \wedge \omega_{b}^{c}
$$

the only non-zero ones then are

$$
R_{2}^{1}=-\left(\partial_{1} \partial_{1}+\partial_{2} \partial_{2}\right) \mu d x^{1} \wedge d x^{2}=-\left[\left(\partial_{1} \partial_{1}+\partial_{2} \partial_{2}\right)\right] e^{-2 \mu} e^{1} \wedge e^{2}
$$

and

$$
R_{4}^{3}=-\left(\partial_{3} \partial_{3}+\partial_{4} \partial_{4}\right) \nu d x^{3} \wedge d x^{4}=-\left[\left(\partial_{3} \partial_{3}+\partial_{4} \partial_{4}\right)\right] e^{-2 \nu} e^{3} \wedge e^{4}
$$

We define the curved-index $\gamma$-matrices via

$$
\gamma^{\mu}=\gamma^{a} E_{a}^{\mu}
$$


where $E_{a}^{\mu} e_{\mu}^{b}=\delta_{b}^{a}$. Hence

$$
E_{1}=e^{-\mu} \partial_{1}, E_{2}=e^{-\mu} \partial_{2}, E_{3}=e^{-\nu} \partial_{3}, E_{4}=e^{-\nu} \partial_{4}
$$

We use the flat space $\gamma$-matrices

$$
\begin{aligned}
& \gamma^{1}=\tau_{1} \otimes \sigma_{1}=\left(\begin{array}{cc}
\mathbf{0} & \sigma_{1} \\
\sigma_{1} & \mathbf{0}
\end{array}\right) \quad, \quad \gamma^{2}=\tau_{1} \otimes \sigma_{2}=\left(\begin{array}{cc}
\mathbf{0} & \sigma_{2} \\
\sigma_{2} & \mathbf{0}
\end{array}\right) \\
& \gamma^{3}=\tau_{1} \otimes \sigma_{3}=\left(\begin{array}{cc}
\mathbf{0} & \sigma_{3} \\
\sigma_{3} & \mathbf{0}
\end{array}\right), \gamma^{4}=\tau_{2} \otimes 1=\left(\begin{array}{cc}
\mathbf{0} & -i 1 \\
i 1 & \mathbf{0}
\end{array}\right) \\
& \gamma^{5} \equiv \gamma^{4} \gamma^{1} \gamma^{2} \gamma^{3}=\left(\begin{array}{cc}
1 & \mathbf{0} \\
\mathbf{0} & -1
\end{array}\right)
\end{aligned}
$$

The explicit form of the Dirac equation thus becomes

$$
\not D_{A} \psi \equiv \gamma^{a} E_{a}^{\mu}\left(\partial_{\mu}+i A_{\mu}+\frac{1}{8} \omega_{\mu}^{b c}\left[\gamma_{b}, \gamma_{c}\right) \psi=0\right.
$$

while (2) reduces to the three equations

$$
\begin{gathered}
2 F_{12}^{+}=\partial_{1} A_{2}-\partial_{2} A_{1}+\left(\partial_{3} A_{4}-\partial_{4} A_{3}\right) e^{2(\mu-\nu)}=e^{2 \mu}\left(\left|\psi_{1}\right|^{2}-\left|\psi_{2}\right|^{2}\right) \\
\bar{\psi}_{1} \psi_{2}+\bar{\psi}_{2} \psi_{1}=i\left(\bar{\psi}_{2} \psi_{1}-\bar{\psi}_{1} \psi_{2}\right)=0
\end{gathered}
$$

since $F_{13}=F_{24}=F_{23}=F_{14}=0$. In component form, (12) reads $e^{-\nu}\left(\partial_{3}+i A_{3}+i \partial_{4}-A_{4}+\frac{1}{2} \partial_{3} \nu+\frac{i}{2} \partial_{4} \nu\right) \psi_{1}+e^{-\mu}\left(\partial_{1}+i A_{1}-i \partial_{2}+A_{2}+\frac{1}{2} \partial_{1} \mu-\frac{i}{2} \partial_{2} \mu\right) \psi_{2}=0$

$e^{-\mu}\left(\partial_{1}+i A_{1}+i \partial_{2}-A_{2}+\frac{1}{2} \partial_{1} \mu+\frac{i}{2} \partial_{2} \mu\right) \psi_{1}+e^{-\nu}\left(-\partial_{3}-i A_{3}+i \partial_{4}-A_{4}-\frac{1}{2} \partial_{3} \nu+\frac{i}{2} \partial_{4} \nu\right) \psi_{2}=0$

Equation (14) shows we must set either $\psi_{1}$ or $\psi_{2}$ equal to zero. Let us start with $\psi_{2}=0$. Then $(13)$ becomes

$$
\left(\partial_{1} A_{2}-\partial_{2} A_{1}\right) e^{-2 \mu}+\left(\partial_{3} A_{4}-\partial_{4} A_{3}\right) e^{-2 \nu)}=\left|\psi_{1}\right|^{2}
$$


Now, the first term in (17) is a function of $\left(x^{1}, x^{2}\right)$ and the second of $\left(x^{3}, x^{4}\right)$. The simplest choice for the RHS compatible with this splitting is

$$
\psi_{1}=\zeta\left(x^{1}, x^{2}\right)+i \eta\left(x^{3}, x^{4}\right)
$$

where $\zeta$ and $\eta$ are real functions. Putting (18) in (15) and (16) then gives

$$
\left[i A_{3}-A_{4}+\left(\frac{1}{2} \partial_{3} \nu+\frac{i}{2} \partial_{4} \nu\right)\right] \zeta=0
$$

or

$$
A_{3}=-\frac{1}{2} \partial_{4} \nu, \quad A_{4}=\frac{1}{2} \partial_{3} \nu
$$

and hence

$$
\left(\partial_{3}+i \partial_{4}\right) \eta=0
$$

Now since $\eta$ is real by (18), (21) does not mean $\eta$ is a holomorphic function; in fact, it can only be a real constant. Using (20), we then have

$$
F_{34}=\frac{1}{2}\left(\partial_{3} \partial_{3}+\partial_{4} \partial_{4}\right) \nu
$$

A similar analysis of (16) gives

$$
\begin{gathered}
A_{1}=-\frac{1}{2} \partial_{4} \mu, \quad A_{2}=\frac{1}{2} \partial_{3} \mu \\
\left(\partial_{1}+i \partial_{2}\right) \zeta=0 \\
F_{12}=\frac{1}{2}\left(\partial_{1} \partial_{1}+\partial_{2} \partial_{2}\right) \mu .
\end{gathered}
$$

Thus $\zeta$ is also a real constant. Putting these back in (17), we find

$$
e^{-2 \mu}\left(\partial_{1} \partial_{1}+\partial_{2} \partial_{2}\right) \mu+e^{-2 \nu}\left(\partial_{3} \partial_{3}+\partial_{4} \partial_{4}\right) \nu=2\left(\eta^{2}+\zeta^{2}\right),
$$


or, comparing with (7) and (8),

$$
R=2 R_{12}^{12}+2 R_{34}^{34}=-2\left(\eta^{2}+\zeta^{2}\right)=-2\left|\psi_{1}\right|^{2} .
$$

We thus see that the Ricci curvature $R$ of the manifold $\mathcal{M}_{4}$ is a negative constant and non-singular solutions are allowed. We can satisfy (26) and (27) by setting

$$
\begin{gathered}
e^{-2 \mu}\left(\partial_{1} \partial_{1}+\partial_{2} \partial_{2}\right) \mu=2|\phi|^{2}=-2 R_{12}^{12} \\
e^{-2 \nu}\left(\partial_{1} \partial_{1}+\partial_{2} \partial_{2}\right) \nu=2\left(\left|\psi_{1}\right|^{2}-|\phi|^{2}\right)=-2 R_{34}^{34},
\end{gathered}
$$

where we have introduced another constant $|\phi|$. The options for $\mathcal{M}_{2}^{(1)}$ and $\mathcal{M}_{2}^{(2)}$ are now related to the values of $|\phi|$ and $\left|\psi_{1}\right|$ in the following way:

(i) Both manifolds have constant negative curvature if $\left|\psi_{1}\right|>|\phi| \neq 0$.

(ii) $\mathcal{M}_{2}^{(1)}$ has zero and $\mathcal{M}_{2}^{(2)}$ has negative curvature if $|\phi|=0$.

(iii) $\mathcal{M}_{2}^{(2)}$ has zero and $\mathcal{M}_{2}^{(1)}$ has negative curvature if $|\phi|=\left|\psi_{1}\right|$.

(iv) $\mathcal{M}_{2}^{(1)}$ has constant negative and $\mathcal{M}_{2}^{(2)}$ has constant positive curvature if $\left|\psi_{1}\right|<|\phi|$.

At this point, it is advantageous to go over to the two pairs of dimensionless coordinates $(x, y) \equiv \sqrt{2}|\phi|\left(x^{1}, x^{2}\right)$ and $(s, t) \equiv \sqrt{2}\left(\left|\psi_{1}\right|^{2}-|\phi|^{2}\right)^{1 / 2}\left(x^{3}, x^{4}\right)$ and then to define their complex combinations

$$
z^{1} \equiv x+i y, \quad z^{2} \equiv s+i t
$$

One can now rewrite (28) and (29) as the pair of Liouville equations

$$
4 \partial_{1} \partial_{\overline{1}} \mu=e^{2 \mu}
$$




$$
4 \partial_{2} \partial_{\overline{2}} \nu= \pm e^{2 \nu} \quad\left(\left|\psi_{1}\right| \gtrless|\phi|\right) .
$$

Cases (i) and (iv) are represented by equation (31) combined with the upper and lower sign versions of (32), respectively. For case (ii) we have the pair of equations

$$
\begin{gathered}
\partial_{1} \partial_{\overline{1}} \mu=0, \\
4 \partial_{2} \partial_{\overline{2}} \nu=e^{2 \nu},
\end{gathered}
$$

while case (iii) is described by the pair

$$
\begin{gathered}
4 \partial_{1} \partial_{\overline{1}} \mu=e^{2 \mu}, \\
\partial_{2} \partial_{\overline{2}} \nu=0 .
\end{gathered}
$$

\section{Gauge invariance of the Ansatz:}

Before considering explicit parametrizations of $\mathcal{M}_{2}^{(1)}$ and $\mathcal{M}_{2}^{(2)}$ based on solutions of (31) - (36), let us investigate the effect of the $U(1)$ transformation

$$
\psi_{1} \rightarrow \exp (i \alpha(x, y, s, t)) \psi_{1}
$$

on (15), (16), (17). The transformation (37) is admitted by the RHS of (17), hence one may wonder whether it leads to new and independent solutions through (19), (20) and (23). The answer is that it does not; the transformation

$$
A_{\mu} \rightarrow A_{\mu}-\partial_{\mu} \alpha
$$

accompanying (37) is forced to have the special form

$$
\alpha=\theta(x, y)+\lambda(s, t)
$$


by the SWME; hence the special form of $A_{\mu}$ in equation (田) of the Ansatz is preserved. This can be shown most easily by using the complex combinations $\partial_{s}+i \partial_{t}=2 \partial_{\overline{2}}, \quad A_{s}+i A_{t}=2 A_{\overline{2}}$, etc. After setting $\psi_{2}=0$ and applying (37), equations (15) and (16) become

$$
\left(2 \partial_{\overline{2}}+\partial_{\overline{2}} \nu+2 i A_{\overline{2}}+2 i \partial_{\overline{2}} \alpha\right) \psi_{1}=0
$$

and

$$
\left(2 \partial_{\overline{1}}+\partial_{\overline{1}} \mu+2 i A_{\overline{1}}+2 i \partial_{\overline{1}} \alpha\right) \psi_{1}=0 .
$$

Recalling that $\psi_{1}=\zeta+i \eta$ and $\zeta, \eta$ are both real constants, it follows that

$$
i \partial_{\overline{2}} \alpha=-i A_{\overline{2}}-\frac{1}{2} \partial_{\overline{2}} \nu
$$

and

$$
i \partial_{\overline{1}} \alpha=-i A_{\overline{1}}-\frac{1}{2} \partial_{\overline{1}} \mu
$$

which can only hold if $\alpha$ has the form (39) (recall that since $\alpha$ is real, we cannot add a function holomorphic in $z_{1}$ and $z_{2}$ to it). This is of course because $A_{\overline{1}}=$ $A_{\overline{1}}\left(z_{1}, \overline{z_{1}}\right), \mu=\mu\left(z_{1}, \overline{z_{1}}\right)$ and $A_{\overline{2}}=A_{\overline{2}}\left(z_{2}, \overline{z_{2}}\right), \nu=\nu\left(z_{2}, \overline{z_{2}}\right)$. Thus the Ansatz is only compatible with gauge transformations of the form (39), which corresponds to having separate U(1) fibers over each of the two-manifolds. Interestingly, (42) and (43) partly realize the original version of H. Weyl's "Eichinvarianz" [9], which was meant to fix the form of the electromagnetic coupling by demanding that field equations transform covariantly under local conformal transformations. 


\section{Solutions of the form $\Sigma_{p_{1}} \times \Sigma_{p_{2}}$ :}

4.1 Cases (ii) and (iii): These are described by the equations (33)-(36). Both represent the same solution with one flat surface and one surface of constant negative curvature; they can be transformed ino each other by $\mu \leftrightarrow \nu, z_{1} \leftrightarrow z_{2}$. It will thus suffice to consider, say, only (33) and (34), which we now do.

We may start by taking $\mu$ as the real part of an analytic function $\zeta\left(z_{1}\right)$. The resulting metric for $\mathcal{M}_{2}^{(1)}$ is then of the form

$$
d s^{2}\left(\mathcal{M}_{2}^{(1)}\right)=\exp \left(\zeta\left(z_{1}\right)+\bar{\zeta}\left(\overline{z_{1}}\right)\right) d z_{1} d \overline{z_{1}}
$$

which can be flattened by going over to the coordinate $\tilde{z}_{1}\left(z_{1}\right)=\int \exp \zeta\left(z_{1}\right) d z_{1}$. $\mathcal{M}_{2}^{(1)}$ can then be turned into a flat torus by choosing $\tilde{z}_{1}\left(z_{1}\right)$ as an inverse elliptic function, tesellating the $\tilde{z_{1}}$ plane into parallelograms.

A similar procedure can be effected on the one-sheeted constant negative curvature hyperboloid stereographically projected onto the complex $g_{2}\left(z_{2}\right)$ plane via Liouville's [10] solution to (34), which reads

$$
\nu=\frac{1}{2} \ln \frac{4\left|\frac{d g_{2}}{d z_{2}}\right|^{2}}{\left(1-g_{2} \bar{g}_{2}\right)^{2}}
$$

At this point, $g\left(z_{2}\right)$ is an arbitrary analytic function. We have therefore the familiar Kleinian metric for $\mathcal{M}_{2}^{(2)}$ :

$$
d s^{2}\left(\mathcal{M}_{2}^{(1)}\right)=e^{2 \nu} d z_{2} d \overline{z_{2}}=\frac{d g_{2} d \overline{g_{2}}}{\left(1-g_{2} \overline{g_{2}}\right)^{2}}
$$


One can of course also put the metric into the Poincaré form

$$
d s^{2}\left(\mathcal{M}_{2}^{(2)}\right)=\frac{d f_{2} d \overline{f_{2}}}{\left(I m f_{2}\right)^{2}}
$$

via $g_{2}\left(z_{2}\right)=\frac{\left(f_{2}-i\right)}{\left(f_{2}+i\right)}$ which maps the interior of the circle $\left|g_{2}\left(z_{2}\right)\right|^{2}=1$ to the upper-half $f$-plane $C_{+}$. If one now chooses $f_{2}\left(z_{2}\right)$ as the Fuchsian function used in uniformizing an algebraic function whose Riemann surface has genus $p_{2}$ [1], $C_{+}$ gets tesellated into $4 p_{2}$-gons with geodesic edges and the manifold $\mathcal{M}_{2}^{(2)}$ becomes the Riemann surface $\Sigma_{p_{2}}$ of genus $p_{2}$ when the edges are identified in the standard way described in, say, [12. For explicit parametrizations of Fuchsian functions we refer the reader to $[13$.

To summarize, the 4-manifolds for cases (ii) and (iii) are of the form $\left(\Sigma_{1} \equiv\right.$ $\left.T^{2}\right) \times \Sigma_{p}, p \geq 2$. Setting the first two components of $A_{\mu}$ which are pure gauge equal to zero, the solution for case (ii) becomes

$$
\begin{gathered}
A=\frac{i}{2}\left\{\frac{1}{2} d \ln \left(\frac{d \bar{g}_{2}}{d \bar{z}_{2}} \frac{d z_{2}}{d g_{2}}\right)+\frac{\left(g_{2} d \bar{g}_{2}-\bar{g}_{2} d g_{2}\right)}{\left(1-g_{2} \bar{g}_{2}\right)}\right\} \\
\omega^{34}=-2 A \\
F=-\frac{1}{2} R^{34}=i \frac{d g_{2} \wedge d \bar{g}_{2}}{\left(1-g_{2} \bar{g}_{2}\right)^{2}}, \\
\Psi^{T}=\left(\psi_{1}, 0,0,0\right), \psi_{1}=c s t ., \quad R=R_{a b}^{a b}=-2\left|\psi_{1}\right|^{2} .
\end{gathered}
$$

In order to obtain the corresponding formulae for case (iii) we simply switch the set $\left(\mu=0, \nu=\nu\left(z_{2}, \overline{z_{2}}\right), g_{2}\left(z_{2}\right), \omega_{4}^{3}, R_{4}^{3}\right)$ with $\left(\nu=0, \mu=\mu\left(z_{1}, \overline{z_{1}}\right), g_{1}\left(z_{1}\right), \omega_{2}^{1}, R_{2}^{1}\right)$.

\subsection{Case (i):}


The pair of Liouville equations (31) and (32) (with the upper sign on the RHS) correspond to the 4-manifold $\Sigma_{p_{1}} \times \Sigma_{p_{2}}\left(p_{1}, p_{2} \geq 2\right)$, where the scalar curvature of the Riemann surfaces are $-2|\phi|^{2}$ and $-2\left(\left|\psi_{1}\right|^{2}-|\phi|^{2}\right)$, respectively. The connections and the curvatures are then given by

$$
\begin{gathered}
\omega_{2}^{1}=-i\left\{\frac{1}{2} d \ln \left(\frac{d \bar{g}_{1}}{d \bar{z}_{1}} \frac{d z_{1}}{d g_{1}}\right)+\frac{\left(g_{1} d \bar{g}_{1}-\bar{g}_{1} d g_{1}\right)}{\left(1-g_{1} \bar{g}_{1}\right)}\right\}, \\
\omega_{4}^{3}=-i\left\{\frac{1}{2} d \ln \left(\frac{d \bar{g}_{2}}{d \bar{z}_{2}} \frac{d z_{2}}{d g_{2}}\right)+\frac{\left(g_{2} d \bar{g}_{2}-\bar{g}_{2} d g_{2}\right)}{\left(1-g_{2} \bar{g}_{2}\right)}\right\}, \\
A=-\frac{1}{2}\left(\omega_{2}^{1}+\omega_{4}^{3}\right), \\
R_{2}^{1}=-2 i \frac{d g_{1} \wedge d \bar{g}_{1}}{\left(1-g_{1} \bar{g}_{1}\right)^{2}}, \quad R_{4}^{3}=-2 i \frac{d g_{2} \wedge d \bar{g}_{2}}{\left(1-g_{2} \bar{g}_{2}\right)^{2}}, \\
F=i \frac{d g_{1} \wedge d \bar{g}_{1}}{\left(1-g_{1} \bar{g}_{1}\right)^{2}}+i \frac{d g_{2} \wedge d \bar{g}_{2}}{\left(1-g_{2} \bar{g}_{2}\right)^{2}}=-\frac{1}{2}\left(R_{2}^{1}+R_{4}^{3}\right),
\end{gathered}
$$

\subsection{Case (iv):}

This is based on (31) and the lower (negative) sign on the RHS of (32). The local form of the solution of (32) is then

$$
\nu=\frac{1}{2} \ln \frac{4\left|\frac{d g_{2}}{d z_{2}}\right|^{2}}{\left(1+g_{2} \bar{g}_{2}\right)^{2}},
$$

which means $\mathcal{M}_{2}^{(2)}=S^{2}$ with radius $\left(\left.|\phi|^{2}|-| \psi_{1}\right|^{2}\right)^{-1 / 2}$ in the original dimensionless coordinates. As in the other cases, $\mathcal{M}_{2}^{(1)}$ is a surface of constant negative curvature, from which a Riemann surface $\Sigma_{p_{2}} \geq 2$ can again be constructed via tesellation by $4 p_{2}$-gons.

$$
\omega_{2}^{1}=-i\left\{\frac{1}{2} d \ln \left(\frac{d \bar{g}_{1}}{d \bar{z}_{1}} \frac{d z_{1}}{d g_{1}}\right)+\frac{\left(g_{1} d \bar{g}_{1}-\bar{g}_{1} d g_{1}\right)}{\left(1-g_{1} \bar{g}_{1}\right)}\right\}
$$




$$
\begin{gathered}
\omega_{4}^{3}=i\left\{\frac{1}{2} d \ln \left(\frac{d \bar{g}_{2}}{d \bar{z}_{2}} \frac{d z_{2}}{d g_{2}}\right)+\frac{\left(g_{2} d \bar{g}_{2}-\bar{g}_{2} d g_{2}\right)}{\left(1+g_{2} \bar{g}_{2}\right)}\right\}, \\
A=-\frac{1}{2}\left(\omega_{2}^{1}+\omega_{4}^{3}\right), \\
R_{2}^{1}=-2 i \frac{d g_{1} \wedge d \bar{g}_{1}}{\left(1-g_{1} \bar{g}_{1}\right)^{2}}, \quad R_{4}^{3}=+2 i \frac{d g_{2} \wedge d \bar{g}_{2}}{\left(1+g_{2} \bar{g}_{2}\right)^{2}} \\
F=i \frac{d g_{1} \wedge d \bar{g}_{1}}{\left(1-g_{1} \bar{g}_{1}\right)^{2}}-i \frac{d g_{2} \wedge d \bar{g}_{2}}{\left(1+g_{2} \bar{g}_{2}\right)^{2}}=-\frac{1}{2}\left(R_{2}^{1}+R_{4}^{3}\right) .
\end{gathered}
$$

In spite of the apparent similarity between the solutions of this section and the previous one, there is one very important difference: since $\mathcal{M}_{2}^{(2)}=S^{2}, g_{2}\left(z_{2}\right)$ is not a Fuchsian function; in fact it can only be

$$
g_{2}\left(z_{2}\right)=\frac{a z_{2}+b}{c z_{2}+d}
$$

which represents the most general one-to-one mappings of $S^{2}$ to itself. Finally, it is easy to show that the solutions in all the cases are entirely invariant under transformations of the form

$$
\tilde{g}_{i}=\frac{\alpha g_{i}+\beta}{ \pm \bar{\beta} g_{i}+\bar{\alpha}}, \quad \alpha \bar{\alpha} \mp+\beta \bar{\beta}, \quad i=1,2
$$

where the upper (lower) sign applies in the negative (positive) curvature cases. By this we mean that not only curvatures but even the connection 1-forms $\omega^{a b}$ and $A$ remain unchanged. Thus the case (i) is invariant under $S U(1,1) \times S U(1,1)$, where both $g_{1}\left(z_{1}\right)$ and $g_{2}\left(z_{2}\right)$ are subjected to (64). The cases (ii)-(iii) and (iv) have invariance groups $S U(1,1)$ and $S U(1,1) \times S U(2)$, respectively. When one passes from the Klein form (40) to the Poincaré form (41) of the hyperboloid 
metric, (64) (upper sign) is replaced by

$$
f_{i}=\frac{a f_{i}+b}{c f_{i}+d}, \quad a b-c d=1,
$$

where $a, b, c, d$ are all real numbers. This is of course $S L(2, \mathbf{R})$ rather than $S U(1,1)$. The $S L(2, \mathbf{Z})$ subgroup of $S L(2, \mathbf{R})$ shuffles $4 p$-gons of the tesellation amongst themselves; invariance under this integer subgroup ensures that one has the same solution in each domain.

\section{Topological numbers and the uniqueness of the solution:}

For a 4-dimensional manifold $\mathcal{M}_{4}$, the signature $\sigma$ is given by [14]

$$
\sigma\left(\mathcal{M}_{4}\right)=-\frac{1}{24 \pi^{2}} \int_{\mathcal{M}_{4}} R_{b}^{a} \wedge R_{a}^{b}
$$

For the solutions considered here, the only non-vanishing components of $R_{b c d}^{a}$ are $R_{212}^{1}$ and $R_{434}^{3}$; hence $\sigma$ trivially vanishes in all the cases (i)-(iv).

The Euler characteristic is most easily computed from the Kunneth formula

$$
\chi\left(\mathcal{M}_{4}\right)=\chi\left(\mathcal{M}_{2}^{(1)}\right) \chi\left(\mathcal{M}_{2}^{(2)}\right)=\left(2-2 p_{1}\right)\left(2-2 p_{2}\right) .
$$

Finally, we must evaluate

$$
c_{1}^{2}=\frac{1}{(2 \pi)^{2}} \int_{\mathcal{M}_{4}} F^{2}
$$

where, as in (56) and (62), we can write

$$
F=-\frac{1}{2}\left(R_{2}^{1}+R_{4}^{3}\right)
$$

and then use (67) and the Gauss-Bonnet theorem to obtain

$$
c_{1}^{2}=\frac{1}{2} \chi\left(\mathcal{M}_{4}\right)
$$


Then, using the notation and conventions of [1], the virtual dimension $W$ of the moduli space defined by

$$
W=-\frac{(2 \chi+3 \sigma)}{4}+c_{1}^{2}=0
$$

is seen to vanish. Thus the solutions of the type $\Sigma_{p_{1}} \times \Sigma_{p_{2}}$ are unique up to $U(1)$ transformations in the bundle and conformal changes of the metrics.

\section{A self-dual solution of the Einstein-Maxwell-Dirac equations:}

The similarities between the solutions of the SWME considered so far and the Bertotti-Robinson solution (covariantly constant electromagnetic fields on a product of two two-manifolds) suggest that some of the field configurations found so far may satisfy the Einstein-Maxwell-Dirac field equations

$$
\begin{gathered}
\mathcal{R}_{\mu \nu}-\frac{1}{2} g_{\mu \nu} \mathcal{R}=\kappa\left(T_{\mu \nu}(\text { e.m. })+T_{\mu \nu}(\text { Dirac })\right)+\Lambda g_{\mu \nu}, \\
F_{; \mu}^{\mu \nu}=j_{e l .}^{\nu}=\psi^{\dagger} \gamma^{\nu} \psi \\
\tilde{F}_{; \mu}^{\mu \nu}=k_{\text {mag. }}^{\nu}=\psi^{\dagger} \gamma^{\nu} \gamma^{5} \psi .
\end{gathered}
$$

The Dirac equation, already satisfied by our solutions, (1) completes the system of coupled equations our three interacting fields have to obey. Using a Weyl spinor in Euclidean signature (which is why the usual $\bar{\psi}$ 's are replaced by $\psi^{\dagger}$ 's) immediately gives

$$
\psi^{\dagger} \gamma^{\nu} \psi=\psi^{\dagger} \gamma^{\nu} \gamma^{5} \psi=0
$$

and

$$
T_{\mu \nu}(\text { Dirac })=\frac{i}{2}\left[\psi^{\dagger} \gamma^{\mu} D_{\nu} \psi-\left(D_{\nu} \psi^{\dagger}\right) \gamma^{\mu} \psi\right]=0
$$


because the $\gamma_{\mu}$ are block off-diagonal while the covariant derivative is block diagonal. Taking the trace of both sides of (72) yields

$$
\mathcal{R}=-4 \Lambda,
$$

or, using (27),

$$
\Lambda=\frac{\left|\psi_{1}\right|^{2}}{2}=\text { constant }
$$

showing that the masless monopole condensate provides a cosmological constant.

Now we know that $F_{\mu \nu}$ is covariantly constant for all our SWME solutions, i.e.,

$$
D^{\mu} F_{\mu \nu}=D^{\mu} \tilde{F}_{\mu \nu}=0
$$

and that these are consistent with (73), (74) and (75); we however must still satisfy the by now considerably simplified Einstein field equations

$$
\mathcal{R}_{\mu \nu}-\frac{1}{2} g_{\mu \nu} \mathcal{R}=\kappa T_{\mu \nu}(\text { e.m. })+\Lambda g_{\mu \nu}
$$

This is most easily accomplished by imposing

$$
F_{\mu \nu}=\tilde{F}_{\mu \nu}=\frac{1}{2} \epsilon_{\mu \nu \alpha \beta} F^{\alpha \beta}
$$

which can be seen to hold when

$$
|\phi|^{2}=\frac{1}{2}|\psi|^{2}
$$

by going back to (22), (25), (28) and (29). This means not only $F_{\mu \nu}$ but the whole solution is self-dual in the sense that the manifolds $\mathcal{M}_{2}^{(1)}$ and $\mathcal{M}_{2}^{(2)}$ are identical! 
Furthermore, explicit calculation, both analytical and by using REDUCE, shows that none of our other SWME solutions obeys (72) - (74).

\section{Kähler structure and relation to the Monge-Ampère equations:}

In the complex coordinates $z^{a}(a=1,2)$ the metric $g_{a \bar{b}}$ of $\mathcal{M}_{4}$ can be obtained from the Kähler potential

$$
U=\mu+\nu=\frac{1}{2} \ln \frac{g_{1}^{\prime} \bar{g}^{\prime}{ }_{1}{ }_{2}{\overline{g^{\prime}}}_{2}}{\left(1-g_{1} \bar{g}_{1}\right)^{2}\left(1-g_{2} \bar{g}_{2}\right)^{2}}
$$

through the Kähler form

$$
K=\frac{i}{2} g_{a \bar{b}} d z^{a} \wedge d \bar{z}^{b}=\frac{i}{2} \partial \bar{\partial} U
$$

Now it is known [14] that if a Kähler potential $\tilde{U}$ satisfies the Monge-Ampère equations

$$
(\bar{\partial} \partial \tilde{U}) \wedge(\bar{\partial} \partial \tilde{U})=\exp 2 \tilde{\Lambda} \tilde{U} d \bar{z}^{1} \wedge d z^{1} \wedge d \bar{z}^{2} \wedge d z^{2}
$$

then the metric for $\tilde{U}$ defined via (84) solves Einstein's equations with cosmolog-

ical constant $\tilde{\Lambda}$; in particular, the Weyl tensor is self-dual. The metric in (84) can be related to (85) by choosing

$$
\tilde{U}=\frac{1}{2 \Lambda}(2 U-3 \ln 2)
$$

Note that $\tilde{\Lambda}$ is dimensionless in the dimensionless coordinates used in (83-86); it can of course always be scaled back together with the coordinates to the value $\Lambda=\frac{\left|\psi_{1}\right|^{2}}{2}$ in (78). The Liouville equations (31) and (321) then guarantee (85). 
The Weyl tensor is self-dual in the trivial sense that all its components vanish.

\section{Concluding remarks:}

One might wonder what would have happened had we chosen the other component of the Weyl spinor to vanish in (15) and (16). Going through the formulae, it is not difficult to see that complex variables are replaced by their conjugates, leading to a sign change in the electromagnetic potential, while the metric remains unchanged. Recalling that two components of the Weyl spinor correspond to particles and antiparticles, we see that the new solution is just the charge conjugate of the original one. We now have an antimonopole condensate and the sense of the vortices is reversed.

It is worth emphasizing here some surprisingly general facts about Weyl spinors and $U(1)$ connections on a 4-manifold of Euclidean signature. As noted in section 6 , (75) and (76) automatically hold. If, in addition, one imposes selfduality on the $F_{\mu \nu}$, Einstein's equations are immediately reduced to the defining equation for an Einstein space and, although not explicitly stated by LeBrun, this is what allows one to consider Einstein spaces in a SW setting without having to take Maxwell's equations and matter tensors into account. Since there is nothing else available, the SW equations then force an identification of the cosmological constant with the monopole condensate. The physical picture is astonishing, at least to the author, in that Weyl spinors and self-dual $U(1)$ gauge fields can seemingly live on an Einstein manifold of Euclidean signature without having 
any other effect on the metric whatsoever! Interestingly, this also "saturates" the LHS of (2) by forcing the curvature to be purely self-dual.

\section{Acknowledgements}

I am grateful to S. Akbulut for answering many questions about SeibergWitten theory, to P. Argyres for clarifying a point involving the virtual dimension, and to Y. Nutku for pointing out the connections to the Bertotti-Robinson solution and the Monge-Ampère equations, as well as carrying out the REDUCE check. I thank M. Arık, T. Dereli and R. Güven for useful discussions and S. Nergiz for help with LATEX.

\section{References}

[1] E. Witten, Math. Res. Lett. 1, 769 (1994).

[2] S. Akbulut, Tr. J. of Mathematics 20, 95 (1996) (Proc. of the 4th Gökova Geometry-Topology Conference).

[3] E. Witten, J. Math. Phys. 35, 5101 (1994).

[4] C. LeBrun, in "The Geometric Universe", ed. by S. A. Huggett, L. J. Mason, K. P. Tod, S. T. Tsou and N. M. J. Woodhouse, Oxford, 1998, p. 109.

[5] C. Saclioglu, Phys. Lett. B 454(1999) 315.

[6] N. Seiberg and E. Witten, Nucl. Phys. B435(1995) 129. 
[7] B. Bertotti, Phys. Rev. 116, 1331 (1959).

[8] I Robinson, Bull. Acad. Pol. Sci. 7 , 351 (1959).

[9] H. Weyl, Sitzungber. Preuss. Akad. Berlin, 465 (1918).

[10] J. Liouville, J. Math. Appl. 18, 71 (1853)

[11] L. R. Ford, Automorphic Functions, Chelsea, NY (1951).

[12] Dubrovin, A. T. Fomenko, and S. P. Novikov, Modern Geometry Vol. II , Springer-Verlag, NY (1985).

[13] Z. Nehari, Conformal Mapping, Dover, NY (1952).

[14] T. Eguchi, P. B. Gilkey and A. J. Hanson, Phys. Rep. 66 (1980) 213. 